International Journal of Heat and Mass Transfer, 165, 2021, Elsevier

DOI: $10.1016 /$ j.ijheatmasstransfer.2020.120610

\title{
A revisit of the electro-diffusional theory for the wall shear stress measurement
}

\author{
Jaromir Havlica ${ }^{\mathrm{a}, \mathrm{b}, *}$, David Kramolis ${ }^{\mathrm{b}}$, Florian Huchet ${ }^{\mathrm{c}}$ \\ ${ }^{a}$ Czech Academy of Sciences, Institute of Chemical Process Fundamentals, Rozvojova \\ 2/135, 16502 Prague, Czech Republic \\ ${ }^{b}$ Jan Evangelista Purkyně University in Ústí nad Labem, Faculty of Science, Pasteurova \\ 3632/15, 40096 Ústi nad Labem, Czech Republic \\ ${ }^{c}$ MAST-GPEM, Univ. Gustave Eiffel, IFSTTAR, Fr-44344 Bouguenais, France
}

\begin{abstract}
This article intends to revisit the electro-diffusional theory for the wall shear stress measurement from mass transfer probes of rectangular shape by considering the existence of two components of the wall shear rate (i.e., axial and transversal). General analytical formulas for the effective transfer length and the dimensionless mass transport coefficient were derived as a function of only two parameters: a dimensionless angle of the flow direction, relative to the leading edge of the probe, and the aspect ratio between the width and the length of the strip probe. The correctness of the analytical relations for arbitrary flow direction and the aspect ratio was confirmed by numerical solutions of the transport equation in the convective-diffusive regime. It has also been proved that the differences between the Lévêque solution and the general analytical formula exhibit a significant deviation for a specific range of parameters. In the case of the three-dimensional boundary layers, in addition to the magnitude of the wall shear stress, the direction of the fluid flow in the vicinity of the probe's surface is of paramount importance. Accordingly, a measurement methodology is proposed using two strip probes with different aspect ratios. The resulting equations required to quantify the magnitude of the wall shear rate vector and the dimensionless angle are also derived.
\end{abstract}

\footnotetext{
* Corresponding author

Email address: havlica@icpf.cas.cz (Jaromir Havlica)
} 
Key words: Boundary layer, Lévêque, Electrochemical method, Mass transfer, Wall shear rate

\section{Introduction}

The efficiency of mass and energy transfer between a solid wall and the surrounding fluid depends on the nature of the hydrodynamics boundary layer [1, 2], and the wall shear stress fundamentally influences these transport phe-

5 nomena. However, to obtain information about the spatial changes of velocity using experimental measurements in the viscous sub-layers is not a trivial matter. The inability of the classical techniques to describe fluid dynamics in the near-wall region has led to the development of a group of non-invasive methods based on a heating element or a mass transfer probe flush-mounted to the wall 3. The Electro-Diffusion (ED) technique is one of the essential representatives of these methods.

The ED method measures the wall shear rate in the liquid phase from the mass transfer probes. A potential difference between an anode of a broad surface (usually located far from the measuring area) and the small working electrode acting as a cathode leads to a fast redox reaction. The ion disappearance occurs only on the microprobe active surface, causing the development of the diffusion boundary layer, in which the fast electro-diffusion reaction rate is controlled only by diffusion in the case of the sufficiently large Schmidt number. The sufficiently large Schmidt number guarantees that the diffusion boundary layer thickness is smaller than the width of the viscous sub-layer, and it allows the simplification of a description of the hydrodynamics near the wall by using the assumption of simple shear flow [4]. The Schmidt number is given by the ratio of the liquid viscosity to the diffusion coefficient of the electrolyte ions. Since in the most measurement cases water and ions of ferricyanide are used, the assumption of sufficiently large Schmidt number can be easily fulfilled for small size probes.

The main drawback of the ED method is posttreatment of measured data to find out the wall shear stress from the limiting diffusion current measurements 
on the base of individual assumptions, which may not always be fulfilled. The fundamentals of ED have been addressed in several works [4, 5]. They conclude that ED measurement has to respect the quasi-steady regime of simple shear flow in the viscous sub-layer perpendicular to the strip probe leading edge. In this case, an analytical solution of the convection-diffusion equation, initially derived by Lévêque [6], can be applied. In 1963 Reiss and Hanratty extended the Lévêque solution also to a circular probe [7].

Nonetheless, the Lévêque solution has limited validity for sufficiently large Péclet numbers, where the convection has to outweigh the diffusion in the axial direction significantly. Then axial diffusion flux can be neglected. However, this assumption cannot be used for smaller values of Péclet number [8] Such cases have been studied by the matched asymptotic expansions [11] and 40 integral boundary method [8] with some simplifications for limiting cases or by a combination of numerical solution of convection-diffusion equation and the inverse method with the necessity of an iterative process [12, 13]. The presented results for small Péclet numbers are pointing at a significant deviation to the Lévêque solution [10].

45 Whenever substantial inertial effect is combined with unsteady phenomenon governs the transport processes, wall shear rate fluctuations are occurring [14, [15], and the frequency response of the mass transfer probes must be taken into account [16. If the amplitude of the flow perturbations is relatively small compared to the time-averaged value, the time-variation of the measured electric current respects the temporal fluctuations of the wall shear rate, and a quasisteady state is generally assumed. At more elevated fluctuation rates, it is necessary to take into consideration the frequency response of the mass transfer probes due to the sufficiently large Schmidt number. In these cases, the capacitive effect of the concentration boundary layer acts as a low-pass filter upon the current fluctuations [17], and the assumption of quasi-steady state is erroneous. The correction between wall shear stress fluctuations and measured fluctuations of limiting diffusion current is restored by transfer functions [18, and their uses have been found relevant, especially for turbulent flow measurements [16]. By 
using the transfer function, it is possible to evaluate the phase shift of the measured current signal concerning the imposed wall shear rate variation of a given frequency, the amplitude attenuation, and correct the power spectra obtained from the measurements of near-wall turbulence [19. The transfer functions can provide information on the frequency-domain from a signal analysis procedure at the cost of the loss of the time-domain information. Therefore, some authors, similarly to the small Péclet number cases, have considered using other methods as, for example, the inverse mass transfer method explored firstly by Mao and Hanratty [20], to deal with the probe inertia while preserving the time-resolved ED measurements. This approach has improved the measurements of the amplitude probability distribution and the frequency spectrum of the streamwise component of the fluctuating velocity gradient. Another procedure for sufficiently high frequency fluctuating flow was introduced by Sobolik et al. [21]. Their method corrects the quasi-steady solution by adding a term, derived from the transient response of the ED probe extended by the time derivative of the wall shear rate. It is based on the assumption of similarity of the concentration fields over the probe surface under steady and unsteady flow conditions. This method was verified numerically for small amplitudes of the fluctuation rate 22 ] and experimentally at higher amplitudes of the fluctuation rate 23] showing its relevancy in turbulent flows compared to the transfer function.

Another problem that may interfere with the correct posttreatment of measured data by ED is the direction of fluid flow. For a circular electrode, due to its symmetry, there is no influence of the angle of the liquid flow on the relation between measured limiting current and wall shear stress. This feature simplifies the interpretation of experimental data, but on the other hand, it does not allow more work with information about the direction of the wall shear stress vector. Unlike the circular probes, rectangular probes are sensitive to the flow orientation in both the axial and transverse directions. The expression for the evaluation of the mass transport coefficient for the slanted probe was firstly presented by Sirkar \& Hanratty [24. For that evaluation, knowledge of the direction of flow around the surface probe is required. However, this information is often 

with one strip probe because from obtained data it is possible to evaluate only the magnitude of the wall shear rate vector. Therefore, if additional information about the flow direction is needed, the more-segmented probes have been proposed. Sirkar \& Hanratty [24] developed the chevron-electrode arrangement tive surfaces with an isolated gap, and Tournier \& Py [26] suggested utilizing the pair of twin rectangular probes. The most commonly used two-segmented probes are, so-called 'sandwich probes', are formed by two strips separated in the mean flow direction by a gap created by an insulator [27. The main problem of this type of two-segmented probes lies in poor sensitivity to transverse flow, and their usage is, therefore, often reduced only to detection of reversal flow in low-frequency oscillating flows [12]. Due to unsatisfactory results with two-segmented probes in terms of flow direction evaluation, the three-segment sensors were developed [28]. The three-segment probes can detect the magnitude of wall shear stress vector and flow direction with a precision better than $10^{\circ}$ with appropriate calibration [29]. However, they exhibit problems with stagnation point flow evaluation and are not suitable for the measurement of high frequency fluctuating flow due to the probe inertia [12]. Such latter complex micro-sensors also suffer from manufacturing constraints for implementation, and the diffusion layer approximation in the trailing edge has to be carefully identified.

In the early stages in the 1960s, the ED method was considered as the sole experimental method able to provide relevant insight into the near-wall flow behavior. Measurements in simple geometries such as straight channel were firstly considered for a better understanding of the boundary layer nature in fully developed turbulent flow [5, 7, 30]. Afterward, ED technique usage was expanded to more complex flows such as flows around bypassing bodies [31, creating of stagnation points e.g. flow-through suddenly changing cross-section [32, or the mixing of fluid streams [33, 34. Since the last two decades, the 120 electro-diffusion method has also been extended to the multiphase systems as 
flows through microporous systems [35]. This sudden transition from fundamental to advanced flow characterization may have led to a misunderstanding in the interpretation of the results while considering the hypothesis of the ED method initially introduced sixty years ago.

If the simple geometry as a straight channel is used, the strip probe can be uniquely positioned on the wall so that its leading edge is perpendicular to the flow direction. In many applications [36, 37, the situation is less clear, and the flow direction is also dependent on the input conditions. In such cases, the probe cannot be placed universally in the space so that the liquid flow is always perpendicular to the leading edge, and the existence of transverses flow directions must be taken into account in addition to the main axial component. In such flow conditions, the analytical solution of Lévêque [6], used in most of the research works, is no longer apply.

In that context, this work intends to revisit the ED technique and show the benefit it may have for the scientist community to take into account the existence of two components of the wall shear rate (i.e., axial and transversal) for the interpretation of measured data. For the Lévêque solution users, pitfalls and sources of errors are outlined. In the present paper, we start from the diffusion-convection equation assuming a homogeneous two-dimensional sim140 ple shear flow without the existence of stagnation points in the measured area $\left(u_{x}, u_{y} \neq 0 ; u_{z} \approx 0\right)$. We reduced the general parametric space of convectiondiffusion transport equation into solely two parameters; one corresponding to the aspect ratio between the width and the length of the strip probe, while the second is related to the flow direction relative to the leading edge of the probe. We derived general analytical formulas for the effective transfer length and the dimensionless mass transport coefficient for fluid flow of arbitrary orientation and any aspect ratio of the strip probe. This relation is a generalization of the solution presented by Sirkar \& Hanratty [24] for a slanted strip probe. The results of the analytical solution for the dimensionless mass transport coefficient were confirmed by a numerical solution of the transport equation with $2 \mathrm{D}$ convective flux. Finally, we presented the new methodology of measurement with 
a strip probe concerning the axial and transversal flow directions.

\section{Strip mass transfer probes: measurement of wall shear stress}

The principle of the electro-diffusion method is based on the measurement

$$
\frac{\partial c}{\partial t}+u_{x} \frac{\partial c}{\partial x}+u_{y} \frac{\partial c}{\partial y}+u_{z} \frac{\partial c}{\partial z}=D \frac{\partial^{2} c}{\partial x^{2}}+D \frac{\partial^{2} c}{\partial y^{2}}+D \frac{\partial^{2} c}{\partial z^{2}},
$$

with the following boundary conditions:

$$
\begin{array}{cccl}
x \rightarrow-\infty & \forall y & z>0 & c=c_{\infty} \\
x \in\left\langle 0, L_{x}\right\rangle & y \in\left\langle 0, L_{y}\right\rangle & z=0 & c=c_{w} \\
x<0 \cup x>L_{x} & y<0 \cup y>L_{y} & z=0 & \frac{\partial c}{\partial z}=0 \\
\forall x & \forall y & z \rightarrow \infty & c=c_{\infty}
\end{array}
$$

where $x, y, z$ are Cartesian coordinates, $L_{x}$ is the width of the probe in the $x$ direction, $L_{y}$ is the length of the probe in the $y$-direction, and $\mathbf{u} \equiv\left(u_{x}, u_{y}, u_{z}\right)$ is the velocity vector.

This work is focused on the simple shear flow flowing under an arbitrary angle $\alpha$ over the mass transfer probe of rectangular shape with any aspect ratio $R$. In such cases, in addition to the axial flow $\left(u_{x} \neq 0\right)$, the existence 
of a transverse direction of the velocity must be taken into account $\left(u_{y} \neq 0\right)$. Since the flow near the probe is supposed to be parallel with the wall, and the existence of stagnation points in the measured area is not expected, the convective transport in the $z$-direction is negligible $\left(u_{z} \approx 0\right)$. It is also assumed that the flow is homogeneous over the surface of the probe and the components of the velocity vector $\left(u_{x}, u_{y}\right)$ vary only in the normal direction to the electrode surface as a linear function in the viscous sub-layer:

$$
u_{x}=\frac{\partial u_{x}}{\partial z} z=S_{x} z
$$

180

$$
u_{y}=\frac{\partial u_{y}}{\partial z} z=S_{y} z
$$

where $S_{x}$ and $S_{y}$ are components of the wall shear rate vector. This assumption can be made since the Schmidt number is sufficiently large. It is also expected that the flow regime is in steady state $\left(\frac{\partial c}{\partial t}=0\right)$. Based on the expectations of sufficiently large Péclet numbers, it can also be assumed that mass transport by diffusion mechanism close to the wall is essential only in the normal direction.

Applying the above assumptions to mass transport governing equation, the eq. (2) is reduced to the form:

$$
S_{x} z \frac{\partial c}{\partial x}+S_{y} z \frac{\partial c}{\partial y}=D \frac{\partial^{2} c}{\partial z^{2}} .
$$

For the sake of simplification, the following discussion of mass transport on the strip probe surface is divided into two parts based on spatial orientations between the probe's leading edge and fluid flow. The examined situation is schematically illustrated in figure 1 a. The first conventional case assumes the perpendicular fluid flow across the leading edge of the probe (figure 1p). The second case assumes fluid flows under the general angle $\alpha$ considering the leading probe edge (figure 1 1 ). The first case leads to the Lévêque solution [6] and the second one to the generalized Sirkar and Harantty solution for the slanted strip probe 24. 

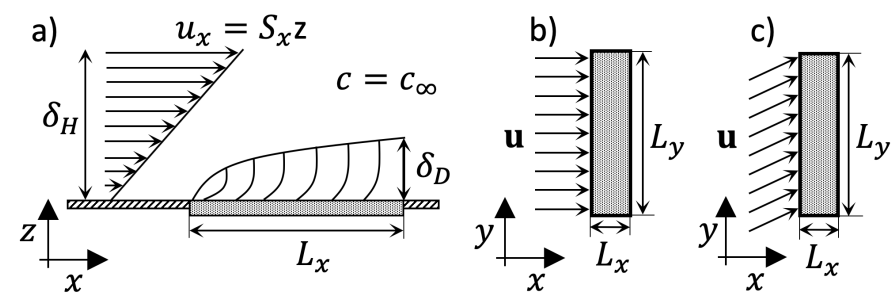

Figure 1: Scheme of the investigated system: a) flow and mass transfer in the near-wall region of the strip probe in $X Z$ plane; b) perpendicular fluid flow over probe surface in $X Y$ plane; c) arbitrary orientation fluid flow over probe surface in $X Y$ plane.

\section{Perpendicular fluid flow: Lévêque solution for strip probe}

If the fluid flow is perpendicular to the leading edge of the probe (see figure 13), equation (6) is simplified into the following form:

$$
S_{x} z \frac{\partial c}{\partial x}=D \frac{\partial^{2} c}{\partial z^{2}}
$$

200 solution [7] such as:

$$
\frac{c-c_{w}}{c_{\infty}-c_{w}}=\frac{1}{\Gamma\left(\frac{4}{3}\right)} \int_{0}^{\eta} \mathrm{e}^{-\eta^{2}} \mathrm{~d} \eta
$$

where $\Gamma$ is the gamma function, and variable $\eta$ is defined as:

$$
\eta=z\left(\frac{S_{x}}{9 D x}\right)^{\frac{1}{3}}
$$

Based on the knowledge of the analytical expression for the concentration field, eq. (8), the relation for the mass transport coefficient on the strip probe was derived by Lévêque [6]:

$$
\bar{k}_{z}=\frac{3}{2 \Gamma\left(\frac{4}{3}\right) 9^{\frac{1}{3}}}\left(\frac{D^{2} S_{x}}{L_{x}}\right)^{\frac{1}{3}}=0.8075\left(\frac{D^{2} S_{x}}{L_{x}}\right)^{\frac{1}{3}} .
$$

In its normalized form, eq. 10 can be rewritten as:

$$
S h_{L_{x}}^{*}=\frac{S h_{L_{x}}}{P e_{s, L_{x}}^{\frac{1}{3}}}=\bar{k}_{z}\left(\frac{L_{x}}{D^{2} S_{x}}\right)^{\frac{1}{3}}=0.8075,
$$


where $S h_{L_{x}}^{*}$ is normalized Sherwood number evaluated for the characteristic length $L_{x}$. For the perpendicular transfer surface case, non-dimensional Sherwood number, $S h_{L_{x}}$, and shear Péclet number, $P e_{s, L_{x}}$, are defined as:

$$
S h_{L_{x}}=\frac{\bar{k}_{z} L_{x}}{D}
$$

$$
P e_{s, L_{x}}=\frac{L_{x}^{2} S_{x}}{D}
$$

Both non-dimensional numbers are also related to the characteristic length corresponding to the width of the probe $L_{x}$.

Using the Faraday relation (1) and the definition of the normalized Sherwood number (11), it is possible to evaluate the wall shear rate $S_{x}$ from measuring current under the limiting diffusion conditions.

\section{The arbitrary orientation of simple shear flow}

For the homogeneous fluid flow, the inlet angle $\alpha$ is related to the components of the wall shear rate vector $\mathbf{S}$ over the whole electrode surface. Therefore, the angle $\alpha$ can be defined in the form of the non-dimensional $\beta$ parameter that is introduced as a square of the ratio of the transverse shear rate to its magnitude, $\|\mathbf{S}\|:$

$$
\beta=\frac{S_{y}^{2}}{S_{x}^{2}+S_{y}^{2}}=\frac{S_{y}^{2}}{\|\mathbf{S}\|^{2}}
$$

and the individual components of the wall shear rate on the wall surface could be re-written as:

$$
\begin{gathered}
S_{x}=\sqrt{1-\beta}\|\mathbf{S}\| ; \\
S_{y}=\sqrt{\beta}\|\mathbf{S}\| .
\end{gathered}
$$

${ }_{225}$ The exact relation between the $\beta$ parameter and inlet angle $\alpha$ is presented in the Appendix A by equations A.18 and A.19.

By substituting equations $(15)$ and $(16)$ into equation (6) one can obtain:

$$
\sqrt{1-\beta}\|\mathbf{S}\| z \frac{\partial c}{\partial x}+\sqrt{\beta}\|\mathbf{S}\| z \frac{\partial c}{\partial y}=D \frac{\partial^{2} c}{\partial z^{2}} .
$$


Equation (17) can be converted into a dimensionless form to simplify the analysis of its solution within the parametric space on the base of the following formulas:

$$
\begin{gathered}
c^{+}=\frac{c-c_{w}}{c_{\infty}-c_{w}} ; x^{+}=\frac{x}{L_{x}} ; y^{+}=\frac{y}{L_{y}} ; z^{+}=\frac{z}{\delta_{D}} ; \\
\delta_{D}=\left(\frac{D l}{\|\mathbf{S}\|}\right)^{\frac{1}{3}} ; R=\frac{L_{x}}{L_{y}},
\end{gathered}
$$
strips oriented in the direction of flow. Since each strip is infinitesimally wide, they can be considered as a separate rectangular probe of length $l(v)$ and width $\mathrm{d} v$. On the base of the Lévêque solution, the mass transfer coefficient for a given narrow strip can be defined according to the equation 10 as follows:

$$
\bar{k}_{z}=0.8075\left(\frac{D^{2}\|\mathbf{S}\|}{l(v)}\right)^{\frac{1}{3}} .
$$


The mean integral value of the mass transfer coefficient, $\bar{k}_{z}$, over the whole probe surface is given by:

$$
\bar{k}_{z}=\frac{1}{L_{x} L_{y}} \int_{S} k_{z} \mathrm{~d} S=\frac{1}{L_{x} L_{y}} \int_{v} k_{z} l(v) \mathrm{d} v=0.8075 \frac{\left(D^{2}\|\mathbf{S}\|\right)^{\frac{1}{3}}}{L_{x} L_{y}} \int_{v} l(v)^{\frac{2}{3}} \mathrm{~d} v .
$$

The eq. 21) is possible rewrite into the form:

$$
\bar{k}_{z}=0.8075\left(\frac{D^{2}\|\mathbf{S}\|}{l_{e}}\right)^{\frac{1}{3}}
$$

where the effective transfer length $l_{e}$ is defined as:

$$
l_{e}^{\frac{1}{3}}=\frac{L_{x} L_{y}}{\int_{v} l(v)^{\frac{2}{3}} \mathrm{~d} v}
$$

The integral in the denominator has to be evaluated for two basic situations:

${ }_{255} \beta \leq \beta_{c}$ and $\beta>\beta_{c}$, where $\beta_{c}$ is the critical parameter $\beta$. The critical parameter $\beta_{c}$ corresponds to a condition where the aspect ratio of the probe equals the ratio of the axial to the transversal velocity components of the flowing fluid over the probe:

$$
\frac{L_{x}}{L_{y}}=\frac{u_{x}}{u_{y}}=\frac{S_{x} z}{S_{y} z}=\frac{\sqrt{1-\beta_{c}}\|\mathbf{S}\| z}{\sqrt{\beta_{c}}\|\mathbf{S}\| z}=\frac{\sqrt{1-\beta_{c}}}{\sqrt{\beta_{c}}} .
$$

It is possible to express $\beta_{c}$ by a simple modification of eq. 24):

$$
\beta_{c}=\frac{L_{y}^{2}}{L_{x}^{2}+L_{y}^{2}}=\frac{1}{R^{2}+1}
$$

For the evaluation of the effective transfer length, the integral in eq. 23 must be divided into three parts, as displayed in figure 2 . In both cases $\left(\beta \leq \beta_{c}\right.$ and $\beta>\beta_{c}$ ), the rectangular probe is divided into two triangles $\mathrm{A}, \mathrm{C}$, and one parallelogram $\mathrm{B}$. The triangles $\mathrm{A}$ and $\mathrm{C}$ are identical from the integration point of view. Based on this division, eq. 23) could be rewritten into the form:

$$
l_{e}^{\frac{1}{3}}=\frac{L_{x} L_{y}}{2 \int_{0}^{v_{s}} l_{t}(v)^{\frac{2}{3}} \mathrm{~d} v+\int_{0}^{v_{c}} l_{p}^{\frac{2}{3}} \mathrm{~d} v}
$$

where $v_{s}$ is the altitude of triangles $\mathrm{A}$ and $\mathrm{C}, v_{c}$ is the altitude of parallelogram $\mathrm{B}$, and $l_{t}(v)$ is the length of strips in both triangles (A and $\mathrm{C}$ ), and $l_{p}$ is the 


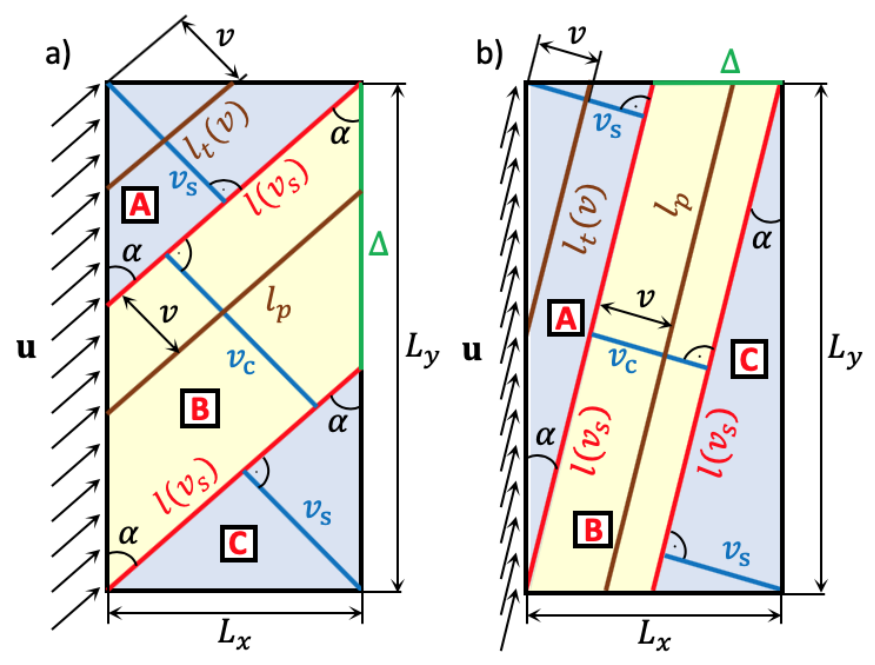

Figure 2: Scheme and description of the rectangular probe to derive the effective transfer length $l_{e}$ for cases: a) $\beta \leq \beta_{c}$ and b) $\beta>\beta_{c}$.

length of strips in the parallelogram. As shown in figure 2, the length $l_{p}$ is independent from the parameter $v$.

By solving the integrals in eq. 26) and subsequent simple adjustments, the 270 equations describing the effective transfer length $l_{e}$ as a function of probe width $L_{x}$, aspect ratio $R$ and parameter $\beta$ can be obtained:

$$
l_{e}^{\frac{1}{3}}=\frac{L_{x} L_{y}}{\int_{v} l(v)^{\frac{2}{3}} \mathrm{~d} v}=\left\{\begin{array}{ll}
\frac{L_{x}^{\frac{1}{3}}}{(1-\beta)^{\frac{1}{6}}\left(1+\frac{R \sqrt{\beta}}{5 \sqrt{1-\beta}}\right)} & \text { for } \beta \leq \beta_{c} \\
\frac{L_{x}^{\frac{1}{3}}}{\beta^{\frac{1}{6}} R^{\frac{1}{3}}\left(1+\frac{\sqrt{1-\beta}}{5 R \sqrt{\beta}}\right)} & \text { for } \beta>\beta_{c}
\end{array} .\right.
$$

A detailed derivation of the equation 27) is given in the Appendix A.

In the case of multidimensional flow, the dimensionless Sherwood and Péclet numbers are defined as follows:

$$
S h_{l}=\frac{\bar{k}_{z} l}{D}
$$

275

$$
P e_{s, l}=\frac{l^{2}\|\mathbf{S}\|}{D}
$$


where $l$ is the characteristic length, and its choice will be discussed below. Now the normalized Sherwood number can be expressed as:

$$
S h_{l}^{*}=\frac{S h_{l}}{P e_{s, l}^{\frac{1}{3}}}=\bar{k}_{z}\left(\frac{l}{D^{2}\|\mathbf{S}\|}\right)^{\frac{1}{3}},
$$

By substituting a combination of eqs. (22) and (27) into equation (30), one can obtain a relation for evaluation of $S h_{l}^{*}$ :

$$
S h_{l}^{*}=0.8075\left(\frac{D^{2}\|\mathbf{S}\|}{l_{e}}\right)^{\frac{1}{3}}\left(\frac{l}{D^{2}\|\mathbf{S}\|}\right)^{\frac{1}{3}}=0.8075\left(\frac{l}{l_{e}}\right)^{\frac{1}{3}},
$$
of normalized Sherwood number is obtained:

$$
S h_{l}^{*}=\left\{\begin{array}{cc}
0.8075(1-\beta)^{\frac{1}{6}}\left(1+\frac{R \sqrt{\beta}}{5 \sqrt{1-\beta}}\right)\left(\frac{l}{L_{x}}\right)^{\frac{1}{3}} \text { for } \beta \leq \beta_{c} \\
0.8075 \beta^{\frac{1}{6}} R^{\frac{1}{3}}\left(1+\frac{\sqrt{1-\beta}}{5 R \sqrt{\beta}}\right)\left(\frac{l}{L_{x}}\right)^{\frac{1}{3}} & \text { for } \beta>\beta_{c}
\end{array} .\right.
$$

The resulting value of the normalized Sherwood number depends on the selection of the characteristic length $l$. It is clear from the relation (31) that if the substitution of the characteristic length $l$ by the effective transfer length $l_{e}$ ${ }_{285}$ is used (see eq. 27) ), the equation (32) is reduced to the form:

$$
S h_{l_{e}}^{*}=\frac{S h_{l_{e}}}{P e_{s, l_{e}}^{\frac{1}{3}}}=\bar{k}_{z}\left(\frac{l_{e}}{D^{2}\|\mathbf{S}\|}\right)^{\frac{1}{3}}=0.8075,
$$

In this case, $S h_{l_{e}}^{*}$ is constant, and it is independent from both parameters $R$ and $\beta$. The value of the normalized Sherwood number is identical to the value of the normalized Sherwood number obtained by the Lévêque solution for the perpendicular probe, compare eqs. (11) and (33). If the effective transfer length $l_{e}$ is known, the magnitude of wall shear rate $\|\mathbf{S}\|$ is evaluated directly from eq. (33) on the base of the evaluated mass transfer coefficient.

Another possibility is to assume that the characteristic length is equal to the width of the probe. Using substitution $l=L_{x}$, the relation 32 has the 


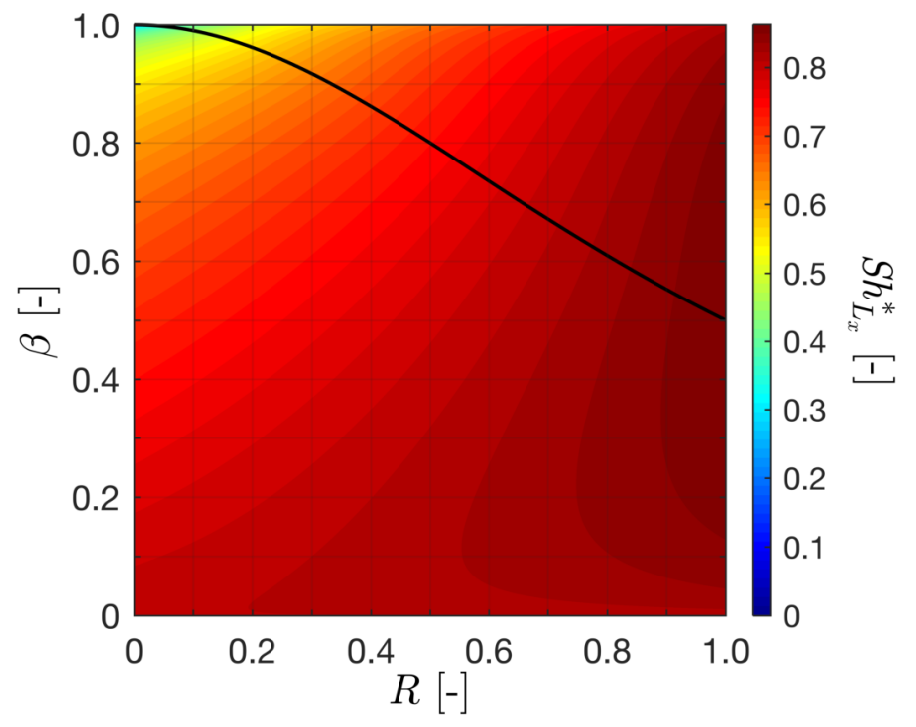

Figure 3: Normalized Sherwood number for length $l=L_{x}$ as function of the parameter $\beta$ and aspect ratio $R$ evaluated from eq. 34. For practical reasons, the aspect ratio is limited to an interval $R \in\langle 0,1\rangle$. The black curve describes the value of the critical $\beta_{c}$ defined by eq. 25.

following form:

$$
S h_{L_{x}}^{*}=\bar{k}_{z}\left(\frac{L_{x}}{D^{2}\|\mathbf{S}\|}\right)^{\frac{1}{3}}=\left\{\begin{array}{ll}
0.8075(1-\beta)^{\frac{1}{6}}\left(1+\frac{R \sqrt{\beta}}{5 \sqrt{1-\beta}}\right) & \text { for } \beta \leq \beta_{c} \\
0.8075 \beta^{\frac{1}{6}} R^{\frac{1}{3}}\left(1+\frac{\sqrt{1-\beta}}{5 R \sqrt{\beta}}\right) & \text { for } \beta>\beta_{c}
\end{array} .\right.
$$

In this situation, the value of the normalized Sherwood number is generally a function of two parameters: probe aspect ratio $R$ and parameter $\beta$. The resulting values of normalized Sherwood number defined by eq. (34) are presented in figure 3. In general, the more the effective transfer length differs from the probe width, the more $S h_{L_{x}}^{*}$ will vary from the derived Lévêque solution, see eq. (31). Figure 3 shows that the minimum value of the normalized Sherwood number can be found for $\beta=1$, and the electrode width is close to zero $(R \rightarrow 0)$. In this situation, normalized Sherwood number is also approaching to zero. Conversely, the 
maximum normalized Sherwood number can be found for the following param-

eters: $\beta=0.5\left(\beta=\beta_{c}\right)$ and $R=1$ (square probe). In this case, $S h_{L_{x}}^{*}=0.8627$. The presented value of $S h_{L_{x}}^{*}$ corresponds to the normalized Sherwood number of a circular probe relative to its diameter derived by Reiss \& Hanratty [7]. It is clear from equation (34) that the Lévêque solution $\left(S h_{L_{x}}^{*}=0.8075\right)$ applies only to the perpendicular transfer surface $(\beta=0)$. In such a case, the effective transfer length is equal to the electrode width $\left(l_{e}=L_{x}\right)$.

During experimental measurements, the direction of fluid flow can be unknown. This fact fundamentally complicates a posttreatment process with the measured data. In the literature, the effective transfer and characteristic length are often substituted by the electrode width $L_{x}$ without taking into account the

15 fluid flow direction. In such cases, it is assumed that the difference between the real value of normalized Sherwood number and the normalized Sherwood number obtained for the perpendicular flow from the Lévêque solution is negligible. But, as shown in figure 3 , the difference between both values of the normalized Sherwood numbers may vary considerably. It applies that the more $S h_{L_{x}}^{*}$ differs ${ }_{20}$ from the value equal to 0.8075 , more significant errors occur whenever the calculation of the wall shear rate is solely based on the Lévêque solution assuming the case of the perpendicular fluid flow over the surface of the probe.

\subsection{Numerical approach}

A second approach, on how to get the appropriate value of the normalized 25 Sherwood number, is based on the calculation of the concentration profiles close to the probe surface by numerical solution of the transport equation (19). In our case, the numerical solution was used, especially to verify the validity of the derived analytical relation for $S h_{L_{x}}^{*}$, see eq. (34). Therefore, the characteristic length $l$ in eq. 19 is defined as the width of the probe $l=L_{x}$. In this case, 330 the eq. 19 is changed to:

$$
z^{+} \frac{\partial c^{+}}{\partial x^{+}}+z^{+} \frac{\sqrt{\beta}}{\sqrt{1-\beta}} \frac{\partial c^{+}}{\partial y^{+}}=\frac{1}{\sqrt{1-\beta}} \frac{\partial^{2} c}{\left(\partial z^{+}\right)^{2}} .
$$

The eq. 35 is dependent only on two parameters: $R$ and $\beta$. Due to the 
transformation into the non-dimensional form, the individual velocity components and the diffusion coefficient have changed, see eq. (35):

$$
\begin{gathered}
u_{x}^{+}=S_{x}^{+} z^{+}=1 . z^{+} ; \\
u_{y}^{+}=S_{y}^{+} z^{+}=\frac{\sqrt{\beta}}{\sqrt{1-\beta}} \cdot z^{+} ; \\
D^{+}=\frac{1}{\sqrt{1-\beta}} \cdot z^{+} .
\end{gathered}
$$

The mass transport in the viscous boundary layer is modeled with a simple shear flow over the wall of the square shape $\left(L_{x}^{+}=1, L_{y}^{+}=1\right)$. A probe is placed on the bottom wall near the inlet. The chosen size of the domain did not affect the shape of the diffusion boundary layer over the probe while minimizing the calculation time. Domain dimensions are in dimensionless quantities. The height of the domain is $H=6$. The length and width are $9 \times 9$. The square electrode has dimensions $1 \times 1$. The following boundary conditions were set for dimensionless concentration: on the probe surface: $c^{+}=0$; inlet: $c^{+}=1$; top wall: $c^{+}=1$; outlet: $\nabla^{+} c^{+}=0$; bottom wall (except the probe): $\frac{\partial c^{+}}{\partial z^{+}}=0$. The finite volume method was used for discretization. A numerical grid was containing $10^{7}$ elements and was non-uniform. Since the greatest change in concentration occurs at the inflow edges of the probe, the numerical grid at the given locations had the highest density to refine the numerical solution. Convection terms were discretized by the UPWIND scheme and diffusion term by the central differencing scheme. For the solution of a system of linear algebraic equations was used smoothSolver with preconditioner method DILU (Simplified diagonal-based incomplete LU smoother for asymmetric matrices). Individual simulations were performed within the OpenFOAM software in which the solver for the numerical solution of equation (35) was programmed.

355 Examples of concentration profiles for different probe aspect ratios $R$ and different parameters $\beta$ are shown in figure 4. In figures 4 a, c, and e, the concentration profiles are presented directly on the bottom wall with the placed probe having a square shape after transformation. In figures $4 \mathrm{~b}, \mathrm{~d}$, and $\mathrm{f}$, the concentration profiles are shown in a plane perpendicular to the bottom wall, 


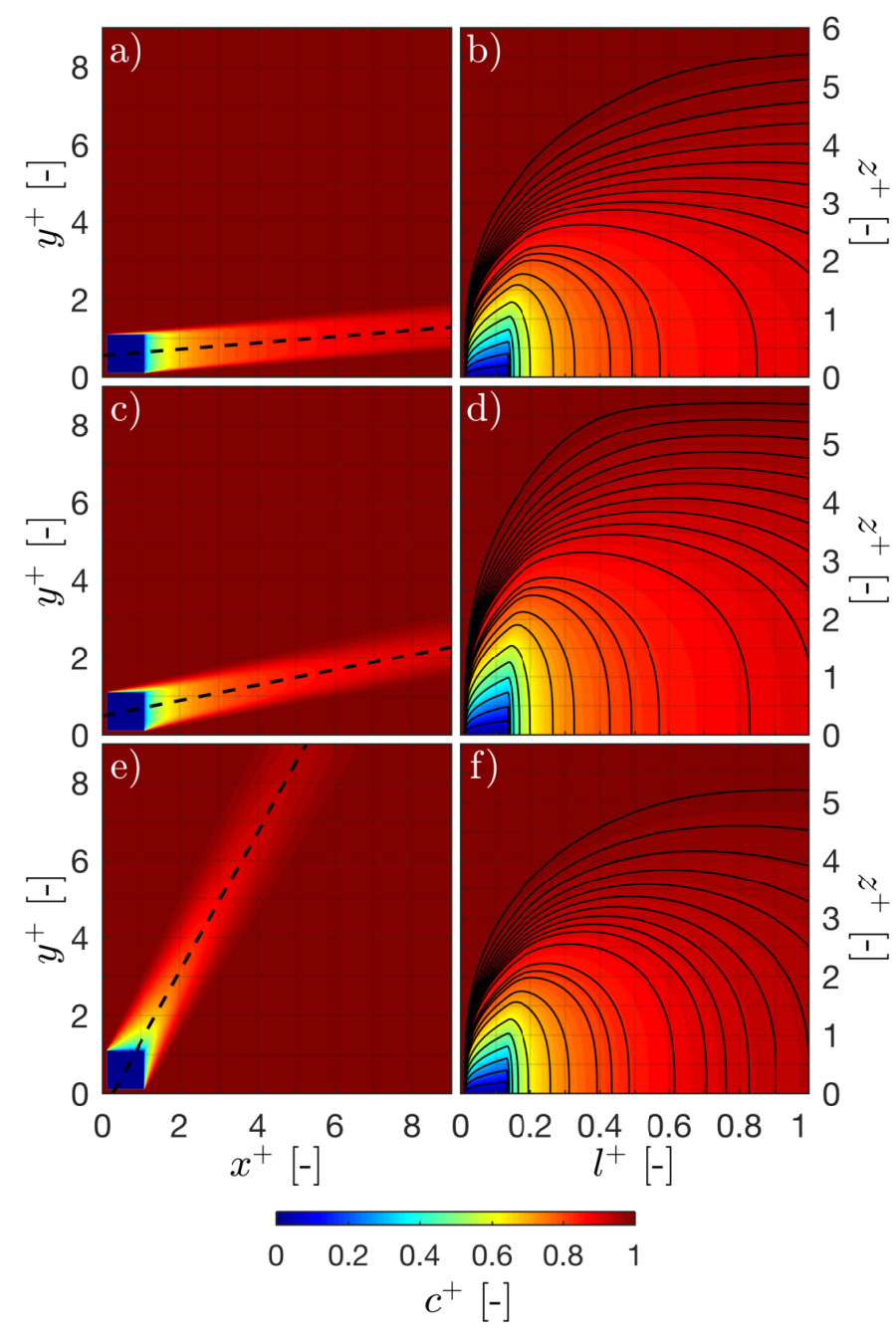

Figure 4: 2D slices of concentration profiles for different probe aspect ratios $R$ and parameters $\beta$ : a), b) $R=0.1, \beta=0.4$; c), d) $R=0.1, \beta=0.8$; e), f) $R=0.9, \beta=0.8$. The first column — the bottom wall with the electrode; the second column — the plane perpendicular to the bottom wall, passing through the center of the electrode and its orientation is in the direction of fluid flow. The black dashed lines in the subplots in the first column represent the position of planes in the second column. Variable $l^{+}$is a relative length of the black dashed line. The black lines are contours. 

fluid flow.

As can be seen in figure 4 the individual concentration profiles depend on the $\beta$ and $R$ parameters. The resulting shape of the concentration profiles depends on the mutual competition between convective and diffuse transport, which could be characterized by the Péclet number:

$$
P e_{s, L_{x}^{+}}=\frac{\left(L_{x}^{+}\right)^{2}\|\mathbf{S}\|}{D^{+}}
$$

whereas the characteristic length corresponds to the width of transformed probe $L_{x}^{+}=1$. By substitution of eqs. 36 - 38 into eq. 39 , the Péclet number is obtained as a function of $\beta$ and $R$ parameters:

$$
P e_{s, L_{x}^{+}}=\sqrt{1-\beta} \sqrt{\frac{1-\beta\left(1-R^{2}\right)}{1-\beta}}=\sqrt{1-\beta\left(1-R^{2}\right)} .
$$

In general, if the Péclet number increases, the importance of convective transport increases compared to diffusion. Therefore, the higher Péclet number results in the reduction of the thickness of the diffusion boundary layer. The increasing the ratio $R$ causes an increase of the Péclet number and a decrease of diffusion boundary layer thickness (compare figure $4 \mathrm{~d} ; P e_{s, L_{x}^{+}}=0.456$ and 4f; $\left.P e_{s, L_{x}^{+}}=0.921\right)$. The increasing the parameter $\beta$ causes a reduction of the 375 Péclet number and, at the same time, the increase of diffusion boundary layer thickness (compare figure $4 \mathrm{~b} ; P e_{s, L_{x}^{+}}=0.777$ and $4 \mathrm{~d} ; P e_{s, L_{x}^{+}}=0.456$ ).

The evaluation of the normalized Sherwood number is computed from the numerical concentration fields around the probe surface, such as:

$$
\begin{aligned}
S h_{L_{x}}^{*}=\frac{S h_{L_{x}}}{P e_{s, L_{x}}} & =\left(\frac{L_{x}^{2}\|\mathbf{S}\|}{D}\right)^{\frac{1}{3}} \frac{L_{x}}{\delta_{D}} \int_{0}^{1} \int_{0}^{1}\left(\frac{\partial c^{+}}{\partial z^{+}}\right) \mathrm{d} x^{+} \mathrm{d} y^{+}= \\
& =\int_{0}^{1} \int_{0}^{1}\left(\frac{\partial c^{+}}{\partial z^{+}}\right) \mathrm{d} x^{+} \mathrm{d} y^{+}=\frac{S h_{L_{x}^{+}}}{P e_{s, L_{x}^{+}}}=S h_{L_{x}^{+}}^{*}
\end{aligned}
$$

As follows from eq. 41 for calculating the normalized Sherwood number, 


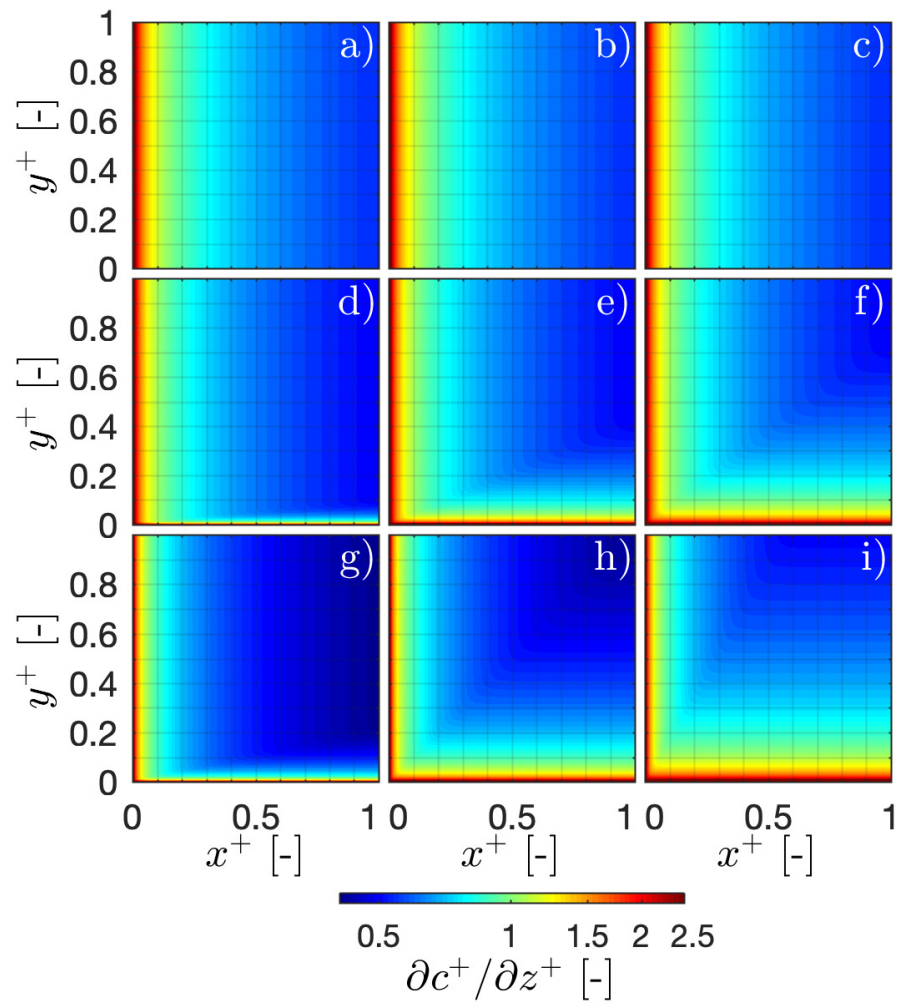

Figure 5: The derivative of concentration in the $z$-direction on the probe surface (logarithmic color bar): the first row $-\beta=0$; the second row $-\beta=0.4$; the third row $-\beta=0.8$; the first column $-R=0.1$; the second column $-R=0.4$; the third column $-R=0.8$.

probe surface is necessary. Examples of these concentration derivatives for different $\beta$ and $R$ parameters only on the surface of the probe are presented in figure 5 The essential fact is that the highest value of the derivative in the normal direction is located around the inlet area according to the $\beta$ parameter value. Then there is a sharp drop in this quantity. Therefore, a very dense numerical grid requires to be used near the inlet edges of the probe. By integrating the derivative of the concentration in the normal direction across the electrode surface based on eq. 41, , a normalized Sherwood number was found. The numerical values of $S h_{L_{x}}^{*}$ coincide with the analytical values obtained from the relation (34) and represented through figure 3 . The relative error between 
the numerical results based on 100 simulations and the analytical solutions, eq. (34), was found, on average, around $10^{-4}$. A further reduction of the error could be achieved by using a higher density numerical grid, especially in the area of liquid inflow on the inner edges of the probe. The agreement between the results proves the correctness of eqs. (31)-(34) for the interpretation of the ED method measurements on the strip probe with any aspect ratio $R$ for a simple shear flow of arbitrary spatial orientation.

4.3. Measurement of wall shear stress with the strip probe for the arbitrary orientation of simple shear flow

Based on Faraday's relation, see eq. (1), the mass transfer coefficient can be obtained from the measured electrical current under limiting diffusion conditions. Unlike the perpendicular case, where the wall shear rate can be calculated directly from eq. 111, in the case of the arbitrary orientation of simple shear flow, eq. (34) cannot be used in this straightforward manner. In addition to the magnitude of the wall shear rate vector, an unknown $\beta$ parameter also occurs in eq. (34). It is necessary to obtain one more independent equation for dimensionless mass transport coefficient to obtain an unambiguous solution $(\beta,\|\mathbf{S}\|)$. This equation can be defined based on a new measurement with a strip probe of other aspect ratios.

It is necessary to make measurements with two probes, namely called $A$ and $B$, with different aspect ratios in such a way that $R_{A}<R_{B}$. During the experiment, it must be guaranteed that the concentration wakes of both probes do not interact with each other, which can be overcome by alternative timeacquiring synchronization of the measurements from both probes or a suitable inter-probe distance. Because of the small dimension of the probe surfaces, the flow field in the near-electrode surfaces is assumed to be homogeneous and, so, the magnitude of the wall shear rate vector and parameter $\beta$ are identical in both cases under a given hydrodynamical flow condition. In that situation, the 
relation (34) can be written as follows:

$$
S h_{L_{x, A}}^{*}\left(\beta, R_{A}\right)=\bar{k}_{z, A}\left(\frac{L_{x, A}}{D^{2}\|\mathbf{S}\|}\right)^{\frac{1}{3}}
$$

420

$$
S h_{L_{x, B}}^{*}\left(\beta, R_{B}\right)=\bar{k}_{z, B}\left(\frac{L_{x, B}}{D^{2}\|\mathbf{S}\|}\right)^{\frac{1}{3}} .
$$

By combining the equations (42) and (43), the unknown $\|\mathbf{S}\|$ is eliminated, and the new relation is only dependent on one unknown, namely the parameter $\beta$ :

$$
\frac{S h_{L_{x, A}}^{*}\left(\beta, R_{A}\right)}{\bar{k}_{z, A} L_{x, A}^{\frac{1}{3}}}=\frac{S h_{L_{x, B}}^{*}\left(\beta, R_{B}\right)}{\bar{k}_{z, B} L_{x, B}^{\frac{1}{3}}} .
$$

As follows from equation (34), the exact relation describing the normalized Sherwood number is dependent on the critical parameter $\beta_{c}$. Because of the difference in $\beta_{c}$ values for both measurement sets, the equation (44) can occur in the following three forms:

$$
f(\beta)= \begin{cases}\frac{R_{A} f_{1}+(1-\beta)^{\frac{1}{6}}}{L_{x, A}^{\frac{1}{3}} \bar{k}_{z, A}}-\frac{R_{B} f_{1}+(1-\beta)^{\frac{1}{6}}}{L_{x, B}^{\frac{1}{3}} \bar{k}_{z, B}}=0 & \text { for } \quad \beta \leq \beta_{c, A}, \beta_{c, B} \\ \frac{R_{A} f_{1}+(1-\beta)^{\frac{1}{6}}}{L_{x, A}^{\frac{1}{3}} \bar{k}_{z, A}}-\frac{f_{2}+\beta^{\frac{1}{6}} R_{B}}{L_{x, B}^{\frac{1}{3}} \bar{k}_{z, B} R_{B}^{\frac{2}{3}}}=0 & \text { for } \quad \beta_{c, B}<\beta \leq \beta_{c, A}, \\ \frac{f_{2}+\beta^{\frac{1}{6}} R_{A}}{L_{x, A}^{\frac{1}{3}} \bar{k}_{z, A} R_{A}^{\frac{2}{3}}-\frac{f_{2}+\beta^{\frac{1}{6}} R_{B}}{L_{x, B}^{\frac{1}{3}} \bar{k}_{z, B} R_{B}^{\frac{2}{3}}}=0} & \text { for } \beta>\beta_{c, A}, \beta_{c, B}\end{cases}
$$

where

$$
f_{1}=\frac{\sqrt{\beta}}{5(1-\beta)^{\frac{1}{3}}} ; \quad f_{2}=\frac{\sqrt{1-\beta}}{5 \beta^{\frac{1}{3}}} .
$$

The solution of equation 45 corresponds to looking for the root of the function $f(\beta)$. It is a nonlinear continuous monotonic decreasing function with only one real $\beta$ root, which can be obtained by any iterative numerical method for finding roots such as the Newton's method. In general, the bigger the difference between the aspect ratio $R_{A}$ and $R_{B}$, the steeper the function $f(\beta)$ decreases. After obtaining the appropriate value of $\beta$ parameter, the last stage is to calculate the magnitude of the wall shear rate vector $\|\mathbf{S}\|$ from eq. 42. or 43 . 
The purpose of this paper is to revisit the electro-diffusional theory for the measurement of wall shear stress from strip mass transfer probes based on the consideration of the existence of the two components of the wall shear rate (i.e., axial and transversal). Therefore, the two-dimensional simple shear flow flowing under an arbitrary angle over the probe of rectangular shape with any aspect ratio was considered. For this type of uniform flow without stagnation points in the measured area, two general analytical formulas were derived for the effective transfer length and the dimensionless mass transport coefficient. These equations are a generalized solution presented by Sirkar \& Hanratty 24 for a slanted strip probe. The correctness of the electro-diffusional theory revision for interpreting measurement data from the ED method was confirmed by numerical solutions of the non-dimensional convection-diffusion equation. It has been shown that if the characteristic length is substituted by the effective transfer length, the dimensionless mass transport coefficient is constant, and the value of the normalized Sherwood number is identical to that obtained with the Lévêque solution for the perpendicular probe. On the other hand, if the characteristic length is defined as the width of the probe, as commonly assumed, the value of the normalized Sherwood number is a function of two parameters: the aspect ratio $R$ and the non-dimensional angle $\beta$. By using the electro-diffusion method for the arbitrary fluid flow direction, it is also often necessary to evaluate, in addition to the magnitude of the wall shear rate vector, the unknown angle of the fluid flow. Therefore, a new measurement methodology based on the proposed analytical relations is applied for two measuring probes of different aspect ratios.

\section{Acknowledgments}

This work was supported by the Ministry of Health of the Czech Republic (project No. 16-29738A) and by Internal Grant Agency of Jan Evangelista 
Purkyně University in Ústí nad Labem (project No. UJEP-SGS-2018-53-0022).

\section{Appendix A.}

The calculation of the effective transfer length is based in particular on the quantification of integrals in the denominator of the equation 26 for two cases: $\beta \leq \beta_{c}$ and $\beta>\beta_{c}$.

For the case $\beta \leq \beta_{c}$, the following equations for altitudes and lengths of strips are applied, see figure 2 .

$$
\begin{gathered}
v_{s}=L_{x} \cos (\alpha) ; \\
v_{c}=\Delta \sin (\alpha)=\left(L_{y}-\frac{v_{s}}{\sin (\alpha)}\right) \sin (\alpha)=L_{y} \sin (\alpha)-L_{x} \cos (\alpha) ; \\
l_{t}(v)=\frac{v}{\cos (\alpha) \sin (\alpha)} ; \\
l_{p}(v)=\frac{L_{x}}{\sin (\alpha)} ;
\end{gathered}
$$

where $\alpha$ is the angle between the leading edge of the rectangular probe, $L_{y}$, 475 and the velocity vector of fluid flow over the surface of the probe. The integrals in the denominator in relation 26 after applying terms A.1 - A.4 can be solved as follows:

$$
\begin{aligned}
& 2 \int_{0}^{v_{s}} l_{t}(v)^{\frac{2}{3}} \mathrm{~d} v=2 \int_{0}^{L_{x} \cos (\alpha)}\left(\frac{v}{\cos (\alpha) \sin (\alpha)}\right)^{\frac{2}{3}} \mathrm{~d} v=\frac{6}{5} L_{x}^{\frac{5}{3}} \frac{\cos (\alpha)}{\sin (\alpha)^{\frac{2}{3}}} \\
& \int_{0}^{v_{c}} l_{p}(v)^{\frac{2}{3}} \mathrm{~d} v=\int_{0}^{L_{y} \sin (\alpha)-L_{x} \cos (\alpha)}\left(\frac{L_{x}}{\sin (\alpha)}\right)^{\frac{2}{3}} \mathrm{~d} v= \\
&=L_{x}^{\frac{2}{3}} L_{y} \sin (\alpha)^{\frac{1}{3}}-L_{x}^{\frac{5}{3}} \frac{\cos (\alpha)}{\sin (\alpha)^{\frac{2}{3}}}
\end{aligned}
$$

Substituting the solutions of the integrals A.5 and A.6 into the equation for calculating the effective transfer length (26), can be get the following formula:

$$
l_{e}^{\frac{1}{3}}=\frac{L_{x} L_{y}}{\frac{6}{5} L_{x}^{\frac{5}{3}} \frac{\cos (\alpha)}{\sin (\alpha)^{\frac{2}{3}}}+L_{x}^{\frac{2}{3}} L_{y} \sin (\alpha)^{\frac{1}{3}}-L_{x}^{\frac{5}{3}} \frac{\cos (\alpha)}{\sin (\alpha)^{\frac{2}{3}}}} .
$$


Based on simple arithmetic adjustments, the relation A.7 can be simplified into the form:

$$
l_{e}^{\frac{1}{3}}=\frac{L_{x}^{\frac{1}{3}}}{\sin (\alpha)^{\frac{1}{3}}\left(1+\frac{L_{x} \cos (\alpha)}{5 L_{y} \sin (\alpha)}\right)} .
$$

For the case $\beta>\beta_{c}$, the altitude of triangles $v_{s}$, the altitude of the parallelogram $v_{c}$, the length of strips in both triangles $l_{t}(v)$ and the length of strips in the parallelogram $l_{p}$ are defined by relations:

$$
\begin{gathered}
v_{s}=L_{y} \sin (\alpha) ; \\
v_{c}=\Delta \cos (\alpha)=\left(L_{x}-\frac{v_{s}}{\cos (\alpha)}\right) \cos (\alpha)=L_{x} \cos (\alpha)-L_{y} \sin (\alpha) ; \\
l_{t}(v)=\frac{v}{\cos (\alpha) \sin (\alpha)} ; \\
l_{p}(v)=\frac{L_{y}}{\cos (\alpha)} ;
\end{gathered}
$$

The integrals in the denominator in relation 26 after applying terms $A .9$ A.12 can be solved as follows:

$$
\begin{aligned}
2 \int_{0}^{v_{s}} l_{t}(v)^{\frac{2}{3}} \mathrm{~d} v=2 & \int_{0}^{L_{y} \sin (\alpha)}\left(\frac{v}{\cos (\alpha) \sin (\alpha)}\right)^{\frac{2}{3}} \mathrm{~d} v=\frac{6}{5} L_{y}^{\frac{5}{3}} \frac{\sin (\alpha)}{\cos (\alpha)^{\frac{2}{3}}} ; \\
\int_{0}^{v_{c}} l_{p}(v)^{\frac{2}{3}} \mathrm{~d} v & =\int_{0}^{L_{x} \cos (\alpha)-L_{y} \sin (\alpha)}\left(\frac{L_{y}}{\cos (\alpha)}\right)^{\frac{2}{3}} \mathrm{~d} v= \\
& =L_{x} L_{y}^{\frac{2}{3}} \cos (\alpha)^{\frac{1}{3}}-L_{y}^{\frac{5}{3}} \frac{\sin (\alpha)}{\cos (\alpha)^{\frac{2}{3}}}
\end{aligned}
$$

By substitution of the integrals' solutions A.13 and A.14 into the equation for calculating the effective transfer length $(26)$, it is got the following formula now for case $\beta>\beta_{c}$ :

$$
l_{e}^{\frac{1}{3}}=\frac{L_{x} L_{y}}{\frac{6}{5} L_{y}^{\frac{5}{3}} \frac{\sin (\alpha)}{\cos (\alpha)^{\frac{2}{3}}}+L_{x} L_{y}^{\frac{2}{3}} \cos (\alpha)^{\frac{1}{3}}-L_{y}^{\frac{5}{3}} \frac{\sin (\alpha)}{\cos (\alpha)^{\frac{2}{3}}}} .
$$

Based on simple arithmetic adjustments, the relation A.15 described effective transfer length can be simplified into the form:

$$
l_{e}^{\frac{1}{3}}=\frac{L_{y}^{\frac{1}{3}}}{\cos (\alpha)^{\frac{1}{3}}\left(1+\frac{L_{y} \sin (\alpha)}{5 L_{x} \cos (\alpha)}\right)} .
$$


It follows from the definition of beta by eq. (14):

$$
\frac{u_{x}}{u_{y}}=\frac{S_{x} z}{S_{y} z}=\frac{\sqrt{1-\beta}\|\mathbf{S}\| z}{\sqrt{\beta}\|\mathbf{S}\| z}=\frac{\sqrt{1-\beta}}{\sqrt{\beta}}=\frac{\sin (\alpha)}{\cos (\alpha)}=\tan (\alpha)
$$

From the equation (A.17), the relation between the goniometric functions and $\beta$ parameter can be obtained:

500

$$
\begin{gathered}
\sin (\alpha)=\sqrt{1-\beta} ; \\
\cos (\alpha)=\sqrt{\beta} .
\end{gathered}
$$

Substituting formulas (18), A.18) and A.19 into equations describing effective transfer length (A.8) and A.16), it is obtained the resulting equation describing the effective transfer length $l_{e}$ as a function of probe width $L_{x}$, aspect ratio $R$ and $\beta$ parameter:

$$
l_{e}^{\frac{1}{3}}=\frac{L_{x} L_{y}}{\int_{v} l(v)^{\frac{2}{3}} \mathrm{~d} v}=\left\{\begin{array}{ll}
\frac{L_{x}^{\frac{1}{3}}}{(1-\beta)^{\frac{1}{6}}\left(1+\frac{R \sqrt{\beta}}{5 \sqrt{1-\beta}}\right)} & \text { for } \beta \leq \beta_{c} \\
\frac{L_{x}^{\frac{1}{3}}}{\beta^{\frac{1}{6}} R^{\frac{1}{3}}\left(1+\frac{\sqrt{1-\beta}}{5 R \sqrt{\beta}}\right)} & \text { for } \beta>\beta_{c}
\end{array} .\right.
$$

\section{References}

[1] S. S. Bu, J. Yang, Q. T. Dong, Q. W. Wang, Experimental study of flow transitions in structured packed beds of spheres with electrochemical technique., Experimental Thermal and Fluid Science 60 (2015) 106-114. doi:10.1016/j.expthermflusci.2014.09.001.

[2] H. Gelderblom, A. van der Horst, R. Haartsen, J. R., A. A. F. M. C. M., van de Ven, F. N. van de Vosse, Analytical and experimental characterization of a miniature calorimetric sensor in a pulsatile flow., Journal of Fluid Mechanics 666 (2011) 428-444. doi:10.1017/S0022112010004234.

[3] H. A. Stone, Heat mass-transfer from surface-films to shear flows at arbitrary peclet numbers., Physics of Fluids a-Fluid Dynamics 1 (1989) 11111122. doi:10.1063/1.857335. 
[4] T. J. Hanratty, J. A. Campbell, Measurement of wall shear stress., Taylor\&Francis, 1983.

[5] J. E. Mitchell, T. J. Hanratty, A study of turbulence at a wall using an electrochemical wall shear-stress meter., Journal of Fluid Mechanics 26 (1966) 199-221. doi:10.1017/S0022112066001174.

[6] A. M. Lévêque, Les lois de la transmission de chaleur par convection., Annales des Mines 13 (1928) 201-415.

[7] L. P. Reiss, T. J. Hanratty, An experimental study of the unsteady nature of the viscous sublayer., Aiche Journal 9 (2) (1963) 154-160. doi:10.1002/ Aic.690090204.

[8] P. I. Geshev, N. S. Safarova, Angular and transient characteristics of circular electrochemical friction probes., International Journal of Heat and Mass Transfer 42 (1999) 3183-3188. doi:10.1016/S0017-9310(98) 00369-X.

[9] S. C. Ling, Heat transfer from a small isothermal spanwise strip on an insulated boundary., Journal of Heat Transfer-Transactions of the Asme 85 (3) (1963) 230-235. doi:10.1115/1.3686079.

[10] C. G. Phillips, Heat and mass-transfer from a film into steady shear-flow., Quarterly Journal of Mechanics and Applied Mathematics 43 (1990) 135155. doi:10.1093/qjmam/43.1.135.

[11] R. C. Ackerberg, R. D. Patel, S. K. Gupta, Heat-mass transfer to a finite strip at small peclet numbers., Journal of Fluid Mechanics 86 (1978) 49-65. doi:10.1017/S0022112078001007.

[12] M. E. Lamarche-Gagnon, J. Vetel, An inverse problem to assess the twocomponent unsteady wall shear rate., International Journal of Thermal Sciences 130 (2018) 278-288. doi:10.1016/j.ijthermalsci.2018.04.022.

[13] F. Rehimi, F. Aloui, S. Ben Asrallah, L. Doubliez, J. Legrand, Inverse method for electrodiffusional diagnostics of flows., International Journal 
of Heat and Mass Transfer 49 (7-8) (2006) 1242-1254. doi:10.1016/j. ijheatmasstransfer.2005.10.008

[14] R. Mathis, I. Marusic, S. I. Chernyshenko, N. Hutchins, Estimating wallshear-stress fluctuations given an outer region input., Journal of Fluid Mechanics 715 (2013) 163-180. doi:10.1017/jfm.2012.508.

[15] S. A. Martemianov, Statistical theory of turbulent mass transfer in electrochemical systems., Russian Journal of Electrochemistry 53 (10) (2017) 1076-1086. doi:10.1134/S1023193517100081.

[16] C. Deslouis, O. Gil, B. Tribollet, Frequency-response of electrochemical sensors to hydrodynamic fluctuations., Journal of Fluid Mechanics 215 (1990) 85-100. doi:10.1017/S0022112090002567.

[17] G. Fortuna, T. J. Hanratty, Frequency response of boundary layer on wall transfer probes., International Journal of Heat and Mass Transfer 14 (1971) 1499-1507. doi:10.1016/0017-9310(71)90195-5.

[18] V. E. Nakoryakov, A. P. Burdukov, O. N. Kashinsky, P. I. Geshev, Electrodiffusion method of investigation into the local structure of turbulent flows., Gasenko, V. G. Novosibirsk.

[19] J. Tihon, V. Tovchigrechko, V. Sobolik, O. Wein, Electrodiffusion detection of the near-wall flow reversal in liquid films at the regime of solitary waves.,

【 Journal of Applied Electrochemistry 33 (2003) 577-587. doi:10.1023/A: 1024988602276 .

[20] Z. Mao, T. J. Hanratty, Application of an inverse mass-transfer method to the measurement of turbulent fluctuations in the velocity-gradient at

口 the wall., Experiments in Fluids 11 (1) (1991) 65-73. doi:10.1007/ Bf00198433.

[21] V. Sobolik, O. Wein, J. Cermak, Simultaneous measurement of film thickness and wall shear-stress in wavy flow of non-newtonian liquids., Col- 
lection of Czechoslovak Chemical Communications 52 (1987) 913-928. doi:10.1135/cccc19870913

[22] D. M. Wang, J. M. Tarbell, An approximate solution for the dynamicresponse of wall transfer probes., International Journal of Heat and Mass Transfer 36 (18) (1993) 4341-4349. doi:10.1016/0017-9310(93)90119-Q

[23] F. Huchet, P. Legentilhomme, J. Legrand, A. Montillet, J. Comiti, Unsteady flows in milli- and microsystems: analysis of wall shear rate fluctuations., Experiments in Fluids 51 (2011) 597-610. doi:10.1007/ s00348-011-1079-1.

[24] K. K. Sirkar, T. J. Hanratty, Limiting behaviour of turbulent transverse velocity component close to a wall., Journal of Fluid Mechanics 44 (1970) 605-614. doi:10.1017/S002211207000201x

[25] B. Py, J. Gosse, Sur la réalisation d'une sonde polarographique sensible à la vitesse et à la direction de l'écoulement., Comptes Rendus Hebdomadaires Des Seances De L Academie Des Sciences Serie A 269 (1969) 401-405.

[26] C. Tournier, B. Py, Behavior of naturally oscillating 3-dimensional flow around a cylinder., Journal of Fluid Mechanics 85 (1978) 161-186. doi: $10.1017 / \mathrm{S} 0022112078000579$

[27] J. S. Son, T. J. Hanratty, Numerical solution for flow around a cylinder at reynolds numbers of 40,200 and 500., Journal of Fluid Mechanics 35 (1969) 369-386. doi:10.1017/S0022112069001169.

[28] O. Wein, V. Sobolik, Theory of direction sensitive probes for electrodiffusion measurement of wall velocity-gradients., Collection of Czechoslovak Chemical Communications 52 (9) (1987) 2169-2180. doi:10.1135/ ccc19872169.

[29] V. Sobolik, O. Wein, O. Gil, B. Tribollet, 3-segment electrodiffusion probes for measuring velocity-fields close to a wall., Experiments in Fluids 9 (1-2) (1990) 43-48. doi:10.1007/Bf00575334. 
[30] L. P. Reiss, T. J. Hanratty, Measurement of instantaneous rates of mass transfer to a small sink on a wall., Aiche Journal 8 (2) (1962) 245-247. doi:10.1002/aic.690080223.

[31] F. Fadla, A. Graziani, F. Kerherve, R. Mathis, M. Lippert, D. Uystepruyst, L. Keirsbulck, Electrochemical measurements for real-time stochastic reconstruction of large-scale dynamics of a separated flow., Journal of Fluids Engineering-Transactions of the Asme 138 (12) (2016) 121204: 1-8. doi:10.1115/1.4034198.

[32] J. Tihon, V. Penkavova, J. Havlica, M. Simcik, The transitional backwardfacing step flow in a water channel with variable expansion geometry., Experimental Thermal and Fluid Science 40 (2012) 112-125. doi:10.1016/ j.expthermflusci.2012.02.006.

[33] F. Huchet, J. Havlica, P. Legentilhomme, A. Montillet, J. Comiti, J. Tihon, Use of electrochemical microsensors for hydrodynamics study in crossing 【 microchannels., Microfluidics and Nanofluidics 5 (2008) 55-64. doi:10. 1007/s10404-007-0220-1.

[34] N. A. Mouheb, A. Montillet, C. Solliec, J. Havlica, P. Legentilhomme, J. Comiti, J. Tihon, Flow characterization in t-shaped and cross-shaped micromixers., Microfluidics and Nanofluidics 10 (2011) 1185-1197. doi: $10.1007 / \mathrm{s} 10404-010-0746-5$

[35] F. Lesage, N. Midoux, M. A. Latifi, New local measurements of hydrodynamics in porous media., Experiments in Fluids 37 (2004) 257-262. doi:10.1007/s00348-004-0811-5.

[36] M. Altheimer, D. Becker, F. P. D'Aleo, P. R. von Rohr, Flow regime and liquid solid mass transfer investigation in a designed porous structure using electrochemical micro-probes., Chemical Engineering Science 152 (2016)

699-708. doi:10.1016/j.ces.2016.06.066. 
[37] F. Huchet, Comments on the paper "flow regime and liquid-solid mass transfer investigation in a designed porous structure using electrochemical micro-probes" by altheimer et al. (2016), Chemical Engineering Science 163 (2017) 255-256. doi:10.1016/j.ces.2017.01.038. 